\title{
Phase 2 Trial of Talactoferrin in Previously Treated Patients With Metastatic Renal Cell Carcinoma
}

\author{
Eric Jonasch, mo 1 \\ Walter M. Stadler, MD $^{2}$ \\ Ronald M. Bukowski, MD ${ }^{3}$ \\ Teresa G. Hayes, MD, PhD ${ }^{4}$ \\ Atul Varadhachary, MD, PhD ${ }^{5}$ \\ Rajesh Malik, $\mathrm{MD}^{5}$ \\ Robert A. Figlin, ${ }^{6}{ }^{6}$ \\ Sandy Srinivas, $\mathrm{MD}^{7}$

\footnotetext{
${ }^{1}$ Department of Genitourinary Oncology, The University of Texas M. D. Anderson Cancer Center, Houston, Texas.

${ }^{2}$ Department of Clinical Research, University of Chicago Medical Center, Chicago, Illinois.

${ }^{3}$ Department of Medicine, CCF Taussig Cancer Center, CCF Lerner College of Medicine, Case Western Reserve University, Cleveland, Ohio.

${ }^{4}$ Department of Hematology/Oncology, Veterans Affairs Medical Center, Houston, Texas.

${ }^{5}$ Agennix, Inc., Houston, Texas.

${ }^{6}$ Department of Medical Oncology and Therapeutics Research, City of Hope National Medical Center, Duarte, California.

${ }^{7}$ Department of Medical Oncology, Stanford University, Stanford, California.
}

Research funding received from Agennix Inc.

Dr. Bukowski has acted as a consultant and speaker for and received research support from Pfizer, Genentech, Bayer, Onyx, Wyeth, and Novartis.

Drs. Varadhachary and Malik are employed by Agennix Inc., which owns development rights to talactoferrin- $\alpha$.

Dr. Figlin has received research support from Agennix Inc.

Address for reprints: Eric Jonasch, MD, Department of Genitourinary Medical Oncology, The University of Texas M. D. Anderson Cancer Center, 1515 Holcombe Blvd., Unit 1374, Houston, TX 77030; Fax: (713) 563-9409; E-mail: ejonasch@ mdanderson.org

Received January 23, 2008; revision received February 14, 2008; accepted February 19, 2008.
BACKGROUND. Talactoferrin (TLF), a recombinant form of human lactoferrin (hLF), is an immunomodulatory iron-binding glycoprotein first identified in breast milk. Its immunomodulatory functions include activation of natural killer (NK) and lymphokine-activated killer cells and enhancement of polymorphonuclear cells and macrophage cytotoxicity. Studies in animal models have shown promising anticancer activity, and clinical antitumor activity has been observed in nonsmall cell lung cancer and other tumor types. The purpose of the current study was to evaluate the activity and safety of TLF in patients with refractory metastatic renal cell carcinoma (RCC).

METHODS. Forty-four adult patients with progressive advanced or metastatic RCC who had failed prior systemic therapy received oral talactoferrin at a dose of $1.5 \mathrm{~g}$ twice daily on a 12 -week-on 2-week-off schedule. Patients were evaluated for progression-free survival at 14 weeks, overall response rate, and progressionfree and overall survival.

RESULTS. TLF was well tolerated. No significant hematologic, hepatic, or renal toxicities were reported. The study met its predefined target with a 14-week progression-free survival rate of $59 \%$. The response rate was $4.5 \%$. The mMedian progression-free survival was 6.4 months and the median overall survival was 21.1 months.

CONCLUSIONS. TLF is a well-tolerated new agent that has demonstrated preliminary signs of clinical activity. Given the lack of toxicity, the lack of rapid disease progression in this cohort, and the preclinical data on immune activation, a randomized study assessing its effects on disease progression in patients with metastatic RCC is rational. Cancer 2008;113:72-7. ( 2008 American Cancer Society.

KEYWORDS: renal cell carcinoma, talactoferrin, clinical antitumor activity, phase 2 trial, survival.

$\mathbf{R}$ enal cell carcinoma (RCC) affects $>40,000$ patients per year in the U.S. and is responsible for close to 13,000 deaths. ${ }^{1}$ Once metastatic, RCC is difficult to treat, and median survival is between 1 and 2 years. ${ }^{2-4}$ Several different treatment modalities are available for metastatic RCC, including immunotherapy, mTOR inhibitors, and vascular endothelial growth factor (VEGF) pathway-targeted therapies. ${ }^{3-7}$ Although each modality provides some benefit in a subset of patients, complete response is exceedingly rare, and the majority of patients with metastatic RCC die of their disease. Additional therapy is clearly needed.

Talactoferrin is a unique recombinant form of human lactoferrin, an important immunomodulatory protein. Lactoferrin, an 80kilodalton $(\mathrm{kD})$ member of the transferrin family of iron-binding glycoproteins, ${ }^{8}$ is expressed throughout the body and is found in the highest concentrations in breast milk. Lactoferrin is also present 
in immune cells and on all body surfaces exposed to the external environment (eg, intestinal mucosal surfaces). Lactoferrin plays an important role in helping to establish the immune system, including the gutassociated lymphoid tissue (GALT), in infants and is involved in cellular growth and differentiation, antimicrobial defense, antiinflammatory activity, immune modulation, and cancer protection. ${ }^{9-14}$ Talactoferrin is produced in Aspergillus niger, ${ }^{15}$ a filamentous fungus, and is structurally identical to native human lactoferrin in all material respects, differing only in its glycosylation.

After oral administration, talactoferrin is transported into the Peyer patches of the GALT, in which it recruits immature circulating dendritic cells (DCs) bearing tumor antigens to the GALT and induces their maturation. DC maturation in the presence of tumor antigens and lymphoid effector cells induces a strong systemic innate and adaptive immune response mediated by anticancer natural killer (NK) and NK-T cells, ${ }^{1,2}$ and CD8+ lymphocytes and NK-T cells. This results in the activation of lymphocytes in tumor-draining lymph nodes (unpublished data), cellular infiltration of distant tumors, ${ }^{3}$ and tumor-cell death. Talactoferrin is not systemically bioavailable, ${ }^{4,5}$ and mounting the initial immune response in the GALT (away from the primary tumor and using a physiologically important pathway) may help minimize the effect of the cancer's local immunosuppressive defenses.

In phase 1 clinical studies, oral talactoferrin was found to be well tolerated without the occurrence of dose-limiting toxicities, and a maximum tolerated dose could not be defined. ${ }^{16}$ Seven patients with advanced or metastatic RCC who were heavily pretreated were evaluated in a phase $1 \mathrm{~b}$ study. All 7 patients demonstrated tumor shrinkage or a reduction in tumor growth rate, and 4 patients remained progression-free for $>6$ months, with 3 patients receiving talactoferrin for $>2$ years. One patient had a durable partial response with $71 \%$ tumor shrinkage by standard Response Evaluation Criteria in Solid Tumors (RECIST) criteria. ${ }^{17}$

Two double-blind, placebo-controlled phase 2 studies have been conducted in patients with nonsmall cell lung cancer (NSCLC), with both studies meeting their prespecified endpoints. A 110-patient study in previously untreated patients with NSCLC evaluated talactoferrin or placebo in combination with carboplatin and paclitaxel. The talactoferrin arm demonstrated an improvement in response rate with trends toward improvement in progression-free survival (PFS) and overall survival (OS). ${ }^{18} \mathrm{~A}$ second study in patients who had failed 1 or 2 prior regi- mens of chemotherapy enrolled 100 patients who received talactoferrin or placebo in addition to best supportive care. Patients in the talactoferrin arm demonstrated an improvement in OS with trends in improvement noted in PFS. ${ }^{19}$

Based on these encouraging preliminary findings, a phase 2 study in patients with advanced RCC was conducted and is reported herein. A talactoferrin dose of $1.5 \mathrm{~g}$ administered orally twice daily was chosen for this and other phase 2 studies. Primary endpoints included 14-week PFS and response rate.

\section{MATERIALS AND METHODS}

The study was opened at 6 sites and Institutional Review Board (IRB) approval was obtained at each site before the initiation of patient enrollment. Funding for the study was provided by the study sponsor (Agennix, Houston, Tex). All patients signed informed consent as per institutional guidelines. Eligible patients had histologically confirmed metastatic or unresectable RCC with predominantly clear cell histology, and had failed at least 1 systemic therapy. Computed tomography (CT) documentation of disease progression within 9 months of the completion of the most recent therapy was required, and target lesions had to be measurable according to RECIST. This determination was investigator-based. The Karnofsky performance status had to be $>70$, total bilirubin $\leq 1.5 \mathrm{mg} / \mathrm{dL}$, creatinine $\leq 2.0 \mathrm{mg} / \mathrm{dL}$, hemoglobin $\geq 10 \mathrm{~g} / \mathrm{dL}$, neutrophil count $\geq 2000 / \mathrm{mm}^{3}$, lymphocyte count $\geq 800 / \mathrm{mm}^{3}$, platelet count $\geq 100000 / \mathrm{mm}^{3}$, aspartate aminotransferase or alanine aminotransferase $<2.5$ times the institutional upper limit of normal, serum calcium $\leq 11.5 \mathrm{mg} / \mathrm{dL}$, international normalized ratio (INR) $\leq 1.2$, and forced expiratory volume in 1 second (FEV1) or forced vital capacity (FVC) $\geq 60 \%$ of predicted. Patients with brain metastases, active ischemic heart disease, symptomatic congestive heart failure, serious active infection, autoimmune disease, radiotherapy administered within 4 weeks, and other malignancies diagnosed within 5 years (apart from nonmelanoma skin cancer) were excluded.

Patients received recombinant human lactoferrin (rhLF) at a dose of $1.5 \mathrm{mg}$ orally twice daily for 12 consecutive weeks followed by a 2 -week break. A maximum of 2 additional cycles were permitted. The first CT scan was obtained at baseline, followed by scans at Weeks 8, 14, 21, 27, 34, 41, 48, and 55. Radiologic response was assessed by the investigator. Patients were followed for OS for 12 months from the initiation of the study treatment or until the median OS for the study was determined, whichever 
occurred later, but not longer than 18 months after the last patient was enrolled.

The study's coprimary endpoints were to detect a PFS rate of $\geq 40 \%$ at 14 weeks or a $12.5 \%$ response rate, either of which were considered to be clinically significant. In a phase 2 trial of bevacizumab in second-line RCC patients, the placebo arm had a 4month PFS rate of $20 \%^{20}$ and was chosen as the historic reference. A sample size of 40 patients provided $>80 \%$ power to detect an increase in the 14 -week PFS from $20 \%$ to $40 \%$, with 1-tailed $\alpha$ of 0.05 . An early stopping rule stated that the study would be terminated if there were no radiologic responses after the first 20 patients were enrolled, or fewer than $10 \%$ of the first 20 patients were alive and free of disease progression at 14 weeks.

\section{RESULTS}

\section{Patient Characteristics}

Between September 2004 and February 2005, 44 patients were enrolled at 6 sites. All patients were evaluable for toxicity and response; therefore, all data are presented in the intent-to-treat population. Twenty-two patients $(50 \%)$ had an Eastern Cooperative Oncology Group (ECOG) performance status of 0 and the remainder had an ECOG performance status of 1 (Table 1). By revised Memorial Sloan-Kettering Cancer Center criteria for previously treated patients, 27 patients (61\%) were low risk and 17 patients $(39 \%)$ were intermediate risk. The median age was 64 years and 28 patients (64\%) were male. Approximately $80 \%$ of the patients were white. Thirteen patients $(30 \%)$ had received $\geq 3$ prior therapies; $80 \%$ had received cytokines, $61 \%$ had received chemotherapy, and $32 \%$ had received investigational agents, including 7 who received sorafenib, 5 who received ABX-EGF, 2 who received temsirolimus, and 1 who received sunitinib. The median time from first diagnosis to treatment was 35.5 months.

\section{Drug Administration and Tolerability}

Twenty-nine patients (66\%) completed cycle 1 of therapy and 20 patients (46\%) completed 2 cycles. Talactoferrin was well tolerated. Forty-two patients (96\%) reported at least 1 adverse event (AE), and 23 $(52 \%)$ reported at least 1 related AE. Of the related AEs, the most common were fatigue, flatulence, abdominal pain, and diarrhea, although none were $>$ grade 2 in severity (Table 2) (determined according to the Common Toxicity Criteria [CTC] grading system). One case of grade 3 dyspnea was considered to be possibly related to the study drug (Table 2). There were no drug-related serious AEs reported.
TABLE 1

Patient Characteristics

\begin{tabular}{llc}
\hline Patients & & No. (\%) \\
\hline Prior nephrectomy & & $41(93)$ \\
Karnofsky performance status & 0 & $22(50)$ \\
& 1 & $22(50)$ \\
Age, y & Mean & 62 \\
Gender & Median & 64 \\
& Male & $28(64)$ \\
No. of prior systemic therapies & Female & $16(36)$ \\
& 1 & $17(39)$ \\
Prognostic risk & 2 & $14(32)$ \\
& $\geq 3$ & $13(30)$ \\
No. of metastatic sites & Low & $27(61)$ \\
& Intermediate & $17(39)$ \\
Sites of metastatic disease & 1 & $8(18)$ \\
& 2 & $14(32)$ \\
Prior therapies & $\geq 3$ & $22(50)$ \\
& Lung & $29(66)$ \\
& Liver & $9(21)$ \\
& Interleukin-2 & $23(52)$ \\
* Memorial Sloan-Kettering Cancer Center reference. & & $22(50)$ \\
& Interferon & $10(23)$ \\
& Capecitabine & $10(23)$ \\
& Gemcitabine & $3(7)$ \\
& Bevacizumab & $14(32)$ \\
\hline
\end{tabular}

\section{Patient Outcomes}

The 14 -week PFS was 59\% $(P<.0001$ for comparison with $20 \%$ ), meeting a prespecified study endpoint. The response rate was $4.5 \%$, with $70.5 \%$ of patients demonstrating stable disease for at least 8 weeks (Table 3). The median PFS was 6.4 months (1-sided 95\% confidence interval $[95 \% \mathrm{CI}]$ of 4.7$)$. The median OS was 21.1 months (1-sided 95\% CI of 19.5) and the 1 -year survival rate was $77 \%$ (Table 4 ).

The first responder was a 47-year-old woman who underwent surgical resection of a T3bN2M0 RCC in 2003 and who demonstrated pulmonary, retroperitoneal, and bone metastases shortly after surgery. She developed disease progression while receiving interferon therapy. Treatment with talactoferrin was initiated and the patient demonstrated a near-complete resolution of her adrenal metastasis and sclerosis of her bone lesion. There was no evidence of disease recurrence at the time of last follow-up, nearly 2 years from the last dose of talactoferrin. The second responder was a 59-yearold man who was diagnosed in January 2004 with metastatic RCC. He was treated with interleukin-2, with progression of disease occurring during treatment. Treatment with talactoferrin was initiated 3.5 months after the completion of interleukin-2 
TABLE 2

Adverse Events Possibly, Most Likely, or Definitely Related to Study Drug, Noted in $>4 \%$ of the Study Population

\begin{tabular}{llllc}
\hline & \multicolumn{3}{c}{ Patients with $\geq \mathbf{1}$ related adverse event } \\
\cline { 2 - 5 } Adverse events reported as related to talactoferrin & Grade $\mathbf{1}^{*}$ & Grade 2* & Grade 3* & Total \\
\hline Patients with $\geq 1$ related adverse event & & & & $23(52)$ \\
Abdominal distension & $6(14)$ & $2(5)$ & 0 & $8(18)$ \\
Abdominal pain & $4(9)$ & 0 & 0 & $4(9)$ \\
Constipation & $3(7)$ & 0 & 0 & $3(7)$ \\
Diarrhea & $6(14)$ & 0 & 0 & $6(14)$ \\
Dyspepsia & $2(5)$ & 0 & 0 & $2(5)$ \\
Nausea & $4(9)$ & 0 & 0 & $4(9)$ \\
Flatulence & $8(18)$ & $4(9)$ & 0 & $12(27)$ \\
Fatigue & $4(9)$ & $2(5)$ & 0 & $6(14)$ \\
Dyspnea & 0 & $1(2)$ & $1(2)$ & $2(5)$ \\
Anemia & $1(2)$ & $1(2)$ & 0 & $2(5)$ \\
\hline & & & & \\
*Determined according to the Common Toxicity Criteria grading system. & & & & \\
\hline
\end{tabular}

TABLE 3

Primary Response Endpoints

\begin{tabular}{lc}
\hline Response & No. (\%) \\
\hline PR & $2(4.5)$ \\
SD & $31(70.5)$ \\
PD & $11(25.0)$ \\
14-week PFS & $26(59)$ \\
\hline
\end{tabular}

PR indicates partial response; SD, stable disease; PD, progressive disease; PFS, progression-free survival.

therapy and the patient developed a partial response at Week 22 .

After developing disease progression while receiving talactoferrin, 33 patients $(75 \%)$ received at least 1 subsequent therapy, whereas 11 patients $(25 \%)$ did not. Eighteen patients received sorafenib, 9 patients received bevacizumab, and 7 patients received sunitinib.

\section{DISCUSSION}

Over the past few decades, immunomodulatory therapy has shown promise in patients with RCC, but significant advances using this approach have been frustrated by the idiosyncratic response characteristics and significant toxicities. On 1 end of the toxicity spectrum, vaccines have demonstrated immunologic activity in several trials, but have not been demonstrated to provide definitive patient benefit. ${ }^{21}$ Problems include finding immunostimulatory protein epitopes, establishing optimal delivery platforms, and developing reliable immunologic endpoints that approximate clinical benefit. On the other end of the
TABLE 4

Secondary Response Endpoints

\begin{tabular}{ll}
\hline Secondary outcomes & In months \\
\hline Median progression-free survival & 6.4 \\
Median overall survival & 21.1 \\
Favorable risk $(\mathrm{n}=27)$ & Not reached \\
Intermediate risk $(\mathrm{n}=17)$ & 19.5 \\
1-year survival & $77 \%$ \\
\hline
\end{tabular}

toxicity spectrum lie the cytokines. High-dose interleukin-2, which was approved in 1992 by the U.S. Food and Drug Administration for the treatment of metastatic RCC on the basis of a small number of complete responses, is a highly challenging agent to administer and benefits only a few patients who receive it. Once again, consistent predictors of treatment response have by and large eluded investigators, and efforts to prospectively validate predictors of interleukin-2 response continue. A further challenge in developing effective immunotherapy is the absence of laboratory endpoints that correlate with clinical benefit. Investigators are thus never sure how close they are to achieving a meaningful impact on tumor biology.

There is a clear unmet need for effective, welltolerated immunomodulatory agents that can be used either as monotherapy or as adjuncts to chemotherapy, immunotherapy, or antivascular therapies. Oral talactoferrin is an immunomodulatory molecule that has shown promising antitumor activity in preclinical models and in a variety of solid tumors. Talactoferrin's postulated DC activation mechanism, which combines the 2 halves of standard immunotherapeutic approaches (antigen presentation through in-migration of immature DCs and activa- 
tion of the immune system through DC maturation) appears to hold promise in a challenging field. The data from 2 randomized studies in lung cancer patients indicate early evidence of talactoferrin's clinical efficacy in solid tumors, and provide a context with which the data in this article can be interpreted.

To our knowledge, the current study is the first phase 2 study assessing the efficacy of oral talactoferrin in patients with refractory RCC. The patients enrolled on the study were heavily pretreated. The prespecified effect on 14-week PFS was met, with the $59 \%$ observed value being statistically significantly increased over the historic reference of $20 \%(P<$ .0001 ), and greater than the targeted value of $40 \%$. Although the study by Yang et al. ${ }^{20}$ on which the historic PFS was based used World Health Organization (WHO) measurement criteria, a comparison of WHO criteria with RECIST to our knowledge has not demonstrated a consistent difference in outcome measures. $^{22-24}$ Data from contemporary clinical trials were not available when this study was designed. In the randomized trial comparing sorafenib with placebo, after 3 months of treatment, 255 patients receiving sorafenib (57\%) achieved a complete or partial response or stable disease compared with 152 patients receiving placebo $(34 \%){ }^{3}$ A post hoc comparison using these data still shows a statistically significant improvement in the PFS rate when compared with the current study $(P<.001)$.

Responses were noted in 2 patients, but the response rate endpoint was not met. Nevertheless, the observed response rate was consistent with talactoferrin's cytostatic mechanism of action and also was consistent with the response rate observed with other cytostatic agents such as sorafenib and bevacizumab. The results on secondary efficacy endpoints including median PFS (6.4 months) and median OS (21.1 months) compare favorably with those reported in the literature in patients with previously treated RCC. ${ }^{3,20}$ Patient selection could explain these results, although analysis of the patient characteristics does not reveal these patients to be significantly different from those in other phase 2 trials.

A more definitive demonstration of talactoferrin's potential growth inhibitory properties would require a randomized trial. This could, for example, be a randomized discontinuation trial, although combination with other active agents may be more attractive. For example, clinical data with talactoferrin in NSCLC have suggested that talactoferrin can enhance the activity of chemotherapy. The additive effect of chemotherapy could potentially be explained by the presumed larger number of tumor antigens available as a result of increased tumor cell killing by chemo- therapy. The larger repertoire of tumor antigens available to the DCs activated by talactoferrin could subsequently result in a greater activation of downstream effector cells responsible for tumor cell killing.

For RCC, in which to our knowledge classic cytotoxic chemotherapy has not been effective to date, combination with 1 of the newer VEGF pathway-targeted agents is a practical choice. Data suggest that the immunologic response in patients with cancer may be decreased by high levels of circulating VEGF, ${ }^{25}$ and preclinical studies suggest that the addition of talactoferrin to sunitinib resulted in enhanced activity (unpublished data). Combination trials with newer agents has often been challenging because of overlapping toxicities, but the attractive safety profile of talactoferrin makes combination trials more feasible.

In summary, talactoferrin is a novel agent that is well tolerated and has demonstrated evidence of potential clinical activity in RCC. Although patient selection may contribute to the results noted in the current study, randomized trials, including possibly evaluating the combination of talactoferrin with a VEGF pathway-targeted agent, are necessary to validate these observations.

\section{REFERENCES}

1. Jemal A, Siegel R, Ward E, Murray T, Xu J, Thun MJ. Cancer statistics, 2007. CA Cancer J Clin. 2007;57:43-66.

2. Motzer RJ, Bacik J, Murphy BA, Russo P, Mazumdar M. Interferon-alfa as a comparative treatment for clinical trials of new therapies against advanced renal cell carcinoma. J Clin Oncol. 2002;20:289-296.

3. Escudier B, Eisen T, Stadler WM, et al. Sorafenib in advanced clear-cell renal-cell carcinoma. $N$ Engl $\mathrm{J} \mathrm{Med.}$ 2007;356:125-134.

4. Hudes G, Carducci M, Tomczak P, et al. Temsirolimus, interferon alfa, or both for advanced renal-cell carcinoma. N Engl J Med. 2007;356:2271-2281.

5. McDermott DF, Regan MM, Clark JI, et al. Randomized phase III trial of high-dose interleukin-2 versus subcutaneous interleukin-2 and interferon in patients with metastatic renal cell carcinoma. J Clin Oncol. 2005;23:133-141.

6. Motzer RJ, Hutson TE, Tomczak P, et al. Sunitinib versus interferon alfa in metastatic renal-cell carcinoma. $N$ Engl $J$ Med. 2007;356:115-124.

7. Pyrhonen S, Salminen E, Ruutu M, et al. Prospective randomized trial of interferon alfa-2a plus vinblastine versus vinblastine alone in patients with advanced renal cell cancer. J Clin Oncol. 1999;17:2859-2867.

8. Kanyshkova TG, Buneva VN, Nevinsky GA. Lactoferrin and its biological functions. Biochemistry (Mosc). 2001;66: $1-7$.

9. Gahr M, Speer CP, Damerau B, Sawatzki G. Influence of lactoferrin on the function of human polymorphonuclear leukocytes and monocytes. J Leukoc Biol. 1991;49:427433. 
10. Bellamy W, Takase M, Wakabayashi H, Kawase K, Tomita M. Antibacterial spectrum of lactoferricin B, a potent bactericidal peptide derived from the N-terminal region of bovine lactoferrin. J Appl Bacteriol. 1992;73:472-479.

11. Vorland LH. Lactoferrin: a multifunctional glycoprotein. APMIS. 1999;107:971-981.

12. Wright DG, Gallin JI. Secretory responses of human neutrophils: exocytosis of specific (secondary) granules by human neutrophils during adherence in vitro and during exudation in vivo. J Immunol. 1979;123:285-294.

13. Varadhachary A, Wolf JS, Petrak K, et al. Oral lactoferrin inhibits growth of established tumors and potentiates conventional chemotherapy. Int J Cancer. 2004;111:398-403.

14. Spadaro M, Curcio C, Varadhachary A, et al. Requirement for IFN-gamma, CD8+ T lymphocytes, and NKT cells in talactoferrin-induced inhibition of neu+ tumors. Cancer Res. 2007;67:6425-6432.

15. Ward PP, Piddington CS, Cunningham GA, Zhou X, Wyatt $\mathrm{RD}$, Conneely OM. A system for production of commercial quantities of human lactoferrin: a broad spectrum natural antibiotic. Biotechnology (N Y). 1995;13:498-503.

16. Hayes TG, Falchook GF, Varadhachary GR, et al. Phase I trial of oral talactoferrin alfa in refractory solid tumors. Invest New Drugs. 2006;24:233-240.

17. Varadhachary A, Spadaro M, Engelmayer J, et al. Talactoferrin alfa is an anti-cancer agent with activity in renal cell cancer (RCC) patients and a novel immunomodulatory mechanism of action. Proc Am Soc Clin Oncol. 1996; Vol:14648.

18. Wang Y, Raghunadharao D, Raman G, et al. Adding oral talactoferrin to first-line NSCLC chemotherapy safely enhanced efficacy in a randomized trial. Proc Am Soc Clin Oncol. 2006;VOL:7095.
19. Parikh PM, Wang Y, Ranade AA, et al. Oral talactoferrin extends survival in patients with recurrent NSCLC in a randomized placebo-controlled phase 2 trial. Proc Am Soc Clin Oncol. 2007; VOL:7540.

20. Yang JC, Haworth L, Sherry RM, et al. A randomized trial of bevacizumab, an anti-vascular endothelial growth factor antibody, for metastatic renal cancer. $N$ Engl J Med. 2003; 349:427-434.

21. Jocham D, Richter A, Hoffmann L, et al. Adjuvant autologous renal tumour cell vaccine and risk of tumour progression in patients with renal-cell carcinoma after radical nephrectomy: phase III, randomised controlled trial. Lancet. 2004;363:594-599.

22. Therasse P, Arbuck SG, Eisenhauer EA, et al. New guidelines to evaluate the response to treatment in solid tumors. European Organization for Research and Treatment of Cancer, National Cancer Institute of the United States, National Cancer Institute of Canada. J Natl Cancer Inst. 2000; 92:205-216.

23. Prasad SR, Saini S, Sumner JE, Hahn PF, Sahani D, Boland GW. Radiological measurement of breast cancer metastases to lung and liver: comparison between WHO (bidimensional) and RECIST (unidimensional) guidelines. J Comput Assist Tomogr. 2003;27:380-384.

24. Therasse P, Le Cesne A, Van Glabbeke M, Verweij J, Judson I. RECIST vs. WHO: prospective comparison of response criteria in an EORTC phase II clinical trial investigating ET743 in advanced soft tissue sarcoma. Eur J Cancer. 2005; 41:1426-1430.

25. Osada T, Chong G, Tansik R, et al. The effect of anti-VEGF therapy on immature myeloid cell and dendritic cells in cancer patients. Cancer Immunol Immunother. 2008 Jan 10 [Epub ahead of print]. 